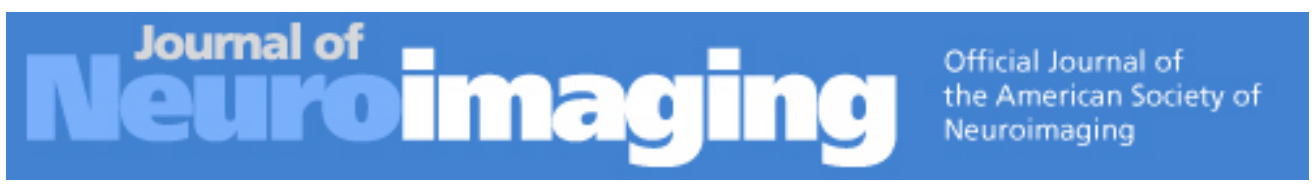

\title{
fMRI in Bell's Palsy: cortical activation is associated with clinical status in the acute and recovery phases
}

\begin{tabular}{|c|c|}
\hline Journal: & Journal of Neuroimaging \\
\hline Manuscript ID & JON-20-5907.R1 \\
\hline Wiley - Manuscript type: & Clinical Investigative Study \\
\hline $\begin{array}{r}\text { Date Submitted by the } \\
\text { Author: }\end{array}$ & $n / a$ \\
\hline Complete List of Authors: & $\begin{array}{l}\text { Calistri, Valentina; Sapienza University of Rome, Human Neurosciences } \\
\text { Mancini, Patrizia; Sapienza University of Rome, Sense Organs } \\
\text { Raz, Eytan; New York University School of Medicine, Radiology } \\
\text { Nicastri, Maria; Sapienza University of Rome, Sense Organs } \\
\text { Tinelli, Emanuele; Sapienza University of Rome, Human Neurosciences } \\
\text { Russo, Francesca; Sapienza University of Rome, Sense Organs } \\
\text { FIORELLI, Marco; Sapienza University of Rome, Human Neurosciences } \\
\text { De Seta, Elio; Sapienza University of Rome, Sense Organs } \\
\text { Carpentieri, Daniele; Umberto I Policlinico di Roma } \\
\text { De Vincentiis, Marco; Sapienza University of Rome, Sense Organs } \\
\text { Caramia, Francesca; Sapienza University of Rome, Human Neurosciences }\end{array}$ \\
\hline Keywords: & fMRI, Bell's Palsy, cerebral cortical activation \\
\hline Subject Area: & $\begin{array}{l}\text { Functional MRI (fMRI) < Magnetic Resonance }(\text { MR) }<\text { Imaging } \\
\text { Techniques < NEUROIMAGING, Peripheral nerve }<\text { Anatomical Region }< \\
\text { NEUROIMAGING }\end{array}$ \\
\hline
\end{tabular}

\section{SCHOLARONE ${ }^{\mathrm{m}}$ Manuscripts}




\section{fMRI in Bell's Palsy: cortical activation is associated with clinical status in the acute}

\section{and recovery phases}

Valentina Calistri ${ }^{1}$, Patrizia Mancini ${ }^{2}$, Eytan Raz $^{3}$, Maria Nicastri ${ }^{2}$, Emanuele Tinelli ${ }^{1}$, Francesca Yoshie Russo $^{2}$, Marco Fiorelli ${ }^{*}$, Elio De Seta ${ }^{2}$, Daniele Carpentieri ${ }^{1}$, Marco De Vincentiis ${ }^{2}$, Francesca Caramia

1. Department of Human Neurosciences, Neuroradiology Unit, Sapienza University, Rome, Italy

2. Department of Sense Organs, Sapienza University, Rome, Italy

3. Department of Radiology, Neuroradiology Unit, New York University School of Medicine, New York, NY, USA

Running title: fMRI in Bell's palsy

Keywords: fMRI; Bell's palsy; cortical activation; 3T

Acknowledgment and Disclosure:

The authors declare that there is no conflict of interest regarding the publication of this article. This work was performed as part of the employment of the authors, University of Rome Sapienza.

\footnotetext{
*Corresponding author

Marco Fiorelli

Department of Human Neurosciences, Neuroradiology Unit

Sapienza University of Rome

Phone: +390649914308 Fax+390649914903

Viale dell'Università, 30 Rome (RM) 00185, Italy

E-mail: marco.fiorelli@uniroma1.it
} 


\begin{abstract}
BACKGROUND: Using functional MRI (fMRI), we explored cortical activation in patients with acute Bell's palsy (BP) and analyzed its correlates with clinical status in the acute phase, and with 6-monthoutcome.

METHODS: 24 right-handed patients with acute BP within 15 days of onset and 24 healthy controls underwent fMRI during performance of unilateral active (hemi-smiling) and passive lip movement tasks with both the paretic and the normal lip. The degree of paresis was evaluated during the acute stage and at the 6-month follow-up using the House-Brackmann (HB) grading scale. Complete recovery was defined as HB grade II or less at the end of the 6-month period. The difference in the HB grade $(\Delta \mathrm{HB})$ between the acute stage and the 6-month follow up was used to evaluate clinical improvement.

RESULTS: There were 24 patients with unilateral acute BP. HB grades ranged from III to VI. At six months, 11 patients (46\%) had completely recovered and $12(50 \%)$ were partially improved. Compared with healthy subjects, BP patients had a significantly greater activation of the frontal areas and the insula ipsilateral to the paretic side. In BP patients, there was an inverse correlation between the activation of the ipsilateral hemisphere when moving the paretic side and the degree of paresis at baseline. An association was also observed between activation and clinical outcome (both complete recovery and $\Delta \mathrm{HB})$.
\end{abstract}

CONCLUSIONS: In patients with Bell's palsy, fMRI may represent a useful tool to predict long-term outcome, guide therapeutic approach, and monitor treatment response. 


\section{Introduction}

Bell's palsy (BP) is a type of peripheral facial palsy that may lead to permanent neurological sequelae. It is characterized by acute, unilateral, partial or complete paralysis of the face, which may be associated with mild pain, numbness, increased sensitivity to sound and altered taste. BP is idiopathic, although in some cases it is suspected to be caused by reactivation of the herpes virus at the geniculate ganglion of the facial nerve. It is the most frequent form of peripheral palsy of the facial nerve, representing about $50-60 \%$ of all etiologies. It is most common in people aged 15 to 40 years, with a variable annual incidence of 8 to 52.8 new cases/100,000 people. ${ }^{1,2}$ The extent of nerve damage determines the degree of recovery. Improvement is gradual and recovery times vary according to severity, age at onset, and the timing and type of therapy..$^{1,3-5}$

Although $70 \%$ of patients experience full recovery within 3 months regardless of treatment, for some patients the symptoms may last longer, and in a few cases they may never completely disappear. ${ }^{6}$ In rare cases the disorder may recur, either on the same or opposite side of the face. ${ }^{7}$ The early detection of patients who will not experience complete recovery is therefore of utmost importance in order to proceed with more aggressive therapies such as facial-nerve surgical decompression..$^{4,8-11}$

Currently prediction of $\mathrm{BP}$ outcome relies on electroneurography together with clinical findings. ${ }^{11-13}$

The brain has the capability to develop adaptive changes and cerebral reorganization that may underlie functional recovery. Previous studies using positron emission tomography and transcranial magnetic stimulation have documented cortical reorganization and its role in recovery in patients with Bell's palsy. ${ }^{14-15}$

Functional magnetic resonance imaging (fMRI) may detect changes in brain activity during execution of a task in pathological conditions and has proved to be a useful tool in the prognosis of central paresis. ${ }^{16-18}$ FMRI has also been applied to peripheral facial nerve palsy and has revealed changes in cortical activation in patients with variable degrees of acute palsy. Cortical reorganization has been 
observed both using task-based fMRI and resting-state fMRI. ${ }^{19-24}$ However the prognostic value of functional cerebral changes in the acute stage of Bell's palsy has not been addressed.

The present study was designed to explore changes in cerebral activation in patients with severe acute unilateral BP and variable degrees of clinical improvement (HB grades I-IV), and to investigate the association between task-based activation, symptom severity, and the degree of clinical improvement over time. 


\section{Methods}

The study was approved by the local ethics committee and all patients gave their written informed consent (ethical committee authorization number \# 29-05-08/1432).

All subjects were recruited from the Ear, Nose and Throat clinic at the Policlinico Umberto I, a teaching hospital affiliated with the University of Rome La Sapienza. The degree of paresis was evaluated according to the House-Brackmann facial nerve grading system $(\mathrm{HB})^{25}$ during the acute phase of BP (within 24 hours and at the time of the fMRI study) and at the 6-month follow-up. Clinical evaluation was performed by the same operator at baseline and follow-up.

To obtain a homogeneous cohort of subjects, the eligibility criteria were as follows: adults aged $>18$ years with unilateral BP; HB grades ranging from III to VI within 48 hours of onset, and righthandedness according to the Edinburgh inventory. ${ }^{26}$ Exclusion criteria included any sign of infective, metabolic, central, or peripheral nervous system disease or temporal bone pathologies and pregnancy. Subjects with secondary facial palsy were also excluded.

A complete recovery was defined as an HB grade of II or less at the end of the 6-month followup period. Improvement of palsy was evaluated as the difference in $\mathrm{HB}$ grade $(\Delta \mathrm{HB})$ between onset and the 6-month follow-up.

All patients were treated within 48 hours of onset with the oral administration of prednisone 1 $\mathrm{mg} / \mathrm{kg}$ for 10 days plus valacyclovir $500 \mathrm{mg}$ q3 for 6 days.

Twenty-four right-handed patients with right (10 subjects, male/female 6/4, median age 56 years, range 24-73) and left (14 subjects, male/female 8/6, median age 50.5 years, range 16-77) BP within 15 days of onset and 24 age and gender-matched healthy controls were prospectively enrolled in our study.

All subjects underwent an MRI scan within 5-15 days of symptom onset (average 10.9 days, $\mathrm{SD}=$ 5 , median=9), which is considered the ideal time frame for intervention. ${ }^{4,27}$

Structural MRI and fMRI were performed in all subjects using a 3T MR system (Verio, 
SIEMENS, Erlangen, Germany) and the following sequences: T2-3D-CISS (TR: 1000 ms, TE: $132 \mathrm{ms,}$ matrix: $384 \times 384$, FOV: $200 \times 200 \mathrm{~mm}$, slice thickness: $0.70 \mathrm{~mm}, 56$ slices per slab, averages: 2), PDT2 weighted (TR: 3320 ms, TE: 10/103 ms, matrix: $384 \times 384$, FOV: $220 \times 220$ mm, slice thickness: 4 mm, gap: 1.2 mm, 50 axial slices), 3D T1-weighted MPRAGE (TR: 2300 ms, TE: 2.98 ms, TI: 900 ms, flip angle: $9^{\circ}$, FOV: $240 \times 256 \mathrm{~mm}, 208$ slices in the sagittal plane, $1 \mathrm{~mm}$ isometric voxel); BOLD T2*_ weighted echo-planar (TR: $3000 \mathrm{~ms}$, TE: $30 \mathrm{~ms}$, flip angle: $90^{\circ}$, thickness: $3 \mathrm{~mm}$, gap: $0.75 \mathrm{~mm}$, matrix: $64 \times 64,36$ axial slices) during the execution of a unilateral active lip task (hemi-smiling, i.e. pulling one side of the mouth upward and backward) executed with both the paretic and normal side.

Subjects were instructed to move the left or right mouth angle up followed by a brief relaxation of facial muscles to regain the starting position. The motor task was performed with a frequency of $1 \mathrm{~Hz}$ for 21 seconds, followed by 21 seconds of rest. Patients were asked to perform the movement with the same effort on both sides, even though it caused minor or no movement on the paretic side. Every subject was trained to execute the task prior to the MRI scan. The presence of mirror movement in the contralateral face was monitored.

Passive movement of the left and right angle of the mouth was also performed. This was obtained using a custom-made plastic hook positioned inside the left or right mouth angle that was moved by an examiner inside the MRI room at the same frequency as active movement ( $1 \mathrm{~Hz}$ for 21 seconds followed by 21 seconds of rest).

FMRI images were analyzed using SPM8 Software (Wellcome Department of Cognitive Neurology, London, UK). Images were first realigned, normalized, and spatially smoothed using a Gaussian kernel of $8 \mathrm{~mm}$. We used the standard echo-planar imaging (EPI) template from SPM to perform spatial normalization. Final voxel size after normalization was $2 \times 2 \times 2 \mathrm{~mm}$. The time series of fMRI images obtained from each participant were analyzed separately. The effects of the experimental paradigm were estimated on a voxel-by-voxel basis using the principles of the general linear model extended to allow the analysis of fMRI data as a time series. The data for each subject was modeled using a boxcar design convolved with the hemodynamic response function chosen to represent the relationship between neuronal activation and blood flow changes in order to obtain single-subject contrast images. 
We recorded mean voxel excursions for all subjects. We did not use motion parameters in the general linear model, but we excluded patients who showed movements larger than $1 \mathrm{~mm}$.

Single-subject contrast images were then used in a second-level analysis according to a random effect model, which included within-group (one-sample t test) and between-group (two-sample $\mathrm{t}$ test) comparisons and regression analysis using the degree of paresis at baseline and clinical outcome (i.e. $\Delta \mathrm{HB}$ at the 6-month follow-up and the presence or absence of complete clinical recovery).

To make patients with right-sided palsy and age and gender-matched healthy controls comparable to patients with left-sided palsy, all images of right-sided palsy patients were flipped along the y-axis prior to analysis. 


\section{Results}

\section{Clinical results}

All patients experienced unilateral facial palsy with HBS grades ranging from III to VI as per inclusion criteria. Assessment of the severity of facial palsy by HB score at baseline (HB0) (range III-VI) and at follow-up (HB6) (range I-VI) is shown in Table 1. Clinical follow-up at 6 months showed improvement in all but one patient, and a complete recovery (HB grade I-II) in 11 patients (46\%).

Table 1. Side and grading of facial nerve palsy.

\begin{tabular}{|c|c|c|c|c|c|}
\hline Patient & Side & Sex & Age & HB-0 & HB-6 \\
\hline$\# 1$ & $\mathrm{~L}$ & $\mathrm{M}$ & 54 & VI & IV \\
\hline$\# 2$ & $\mathrm{~L}$ & M & 18 & IV & NA \\
\hline$\# 3$ & $\mathrm{~L}$ & M & 42 & VI & II \\
\hline$\# 4$ & $\mathrm{R}$ & M & 66 & III & I \\
\hline$\# 5$ & $\mathrm{R}$ & $\mathrm{F}$ & 26 & V & III \\
\hline$\# 6$ & $\mathrm{~L}$ & $\mathrm{~F}$ & 41 & V & II \\
\hline$\# 7$ & $\mathrm{~L}$ & $\mathrm{~F}$ & 75 & VI & V \\
\hline$\# 8$ & $\mathrm{R}$ & $\mathrm{F}$ & 39 & V & III \\
\hline$\# 9$ & $\mathrm{R}$ & $\mathrm{F}$ & 45 & V & I \\
\hline$\# 10$ & $\mathrm{R}$ & $\mathrm{F}$ & 66 & V & I \\
\hline$\# 11$ & $\mathrm{~L}$ & $\mathrm{M}$ & 47 & V & NA \\
\hline$\# 12$ & $\mathrm{R}$ & $\mathrm{M}$ & 46 & III & I \\
\hline$\# 13$ & $\mathrm{~L}$ & $\mathrm{~F}$ & 68 & V & II \\
\hline$\# 14$ & $\mathrm{R}$ & $\mathrm{M}$ & 73 & III & I \\
\hline$\# 15$ & $\mathrm{~L}$ & $\mathrm{~F}$ & 71 & III & II \\
\hline$\# 16$ & $\mathrm{~L}$ & $\mathrm{M}$ & 16 & V & I \\
\hline$\# 17$ & $\mathrm{~L}$ & M & 60 & V & NA \\
\hline
\end{tabular}




$\begin{array}{llllll}\# 18 & \mathrm{~L} & \mathrm{M} & 55 & \mathrm{VI} & \mathrm{VI} \\ \# 19 & \mathrm{~L} & \mathrm{M} & 77 & \mathrm{VI} & \mathrm{V} \\ \# 20 & \mathrm{~L} & \mathrm{~F} & 42 & \mathrm{~V} & \mathrm{NA} \\ \# 21 & \mathrm{R} & \mathrm{M} & 67 & \mathrm{VI} & \mathrm{V} \\ \# 22 & \mathrm{~L} & \mathrm{~F} & 57 & \mathrm{VI} & \mathrm{V} \\ \# 23 & \mathrm{R} & \mathrm{M} & 73 & \mathrm{~V} & \mathrm{IV} \\ \# 24 & \mathrm{R} & \mathrm{M} & 25 & \mathrm{III} & \mathrm{I}\end{array}$

$\mathrm{HB}=$ House-Brackmann score assessing the severity of facial palsy; HB0= House-Brackmann score at baseline (range III-VI); HB6=House-Brackmann score at follow-up (range I-VI); NA= not available; $\mathrm{L}=$ left; $\mathrm{R}=$ right; $\mathrm{M}=$ male; $\mathrm{F}=$ female

\section{Functional MRI analysis}

Both translation and rotation movements were $<1 \mathrm{~mm}$ or $<1^{\circ}$ in all subjects. Therefore, all subjects were included for second-level analysis.

During active motor tasks, the within-group analysis of controls showed activation of areas located on the hemisphere contralateral to the movement and in the bilateral cerebellum (contralateral precentral gyrus and inferior frontal gyrus, contralateral postcentral gyrus, medial frontal gyrus, and bilateral cerebellum; SPM8, cluster-level corrected $\mathrm{p}<0.05$; see Figure 1 and Table 2).

Table 2. Within-group analysis in control subjects during active motor task.

\begin{tabular}{lccccc}
\hline Activation area & \multicolumn{3}{c}{ MNI Coordinates } & $\mathrm{K}$ & $\mathrm{Z}$ \\
\hline & $\mathrm{x}$ & $\mathrm{y}$ & $\mathrm{z}$ & & \\
\hline Contralateral precentral gyrus & 58 & 4 & 10 & 182 & 5.89 \\
& 56 & -2 & 28 & 353 & 5.33 \\
& 32 & -8 & 42 & 4 & 4.79
\end{tabular}




$\begin{array}{lccccr}\text { Contralateral inferior frontal gyrus } & 48 & 4 & 20 & 182 & 5.64 \\ \text { Ipsilateral cerebellum } & -24 & -58 & -28 & 118 & 5.50 \\ \text { Contralateral cerebellum } & 26 & -60 & -26 & 169 & 5.46 \\ \text { Contralateral post-central gyrus } & 52 & -18 & 34 & 353 & 5.18 \\ & & & & & \\ \text { Contralateral medial frontal gyrus } & 10 & -6 & 62 & 3 & 4.80\end{array}$

$\mathrm{MNI}=$ Montreal Neurological Institute and Hospital $\mathrm{p}<0.05 ; \mathrm{K}=$ cluster size; $\mathrm{Z}=$ peak Z-score

In BP patients, the movement of the unaffected side elicited activation involving areas similar to controls (contralateral insula, bilateral superior temporal gyrus, precentral gyrus, and contralateral inferior frontal gyrus, contralateral postcentral gyrus and medial frontal gyrus, bilateral cerebellum; SPM8, cluster-level corrected $\mathrm{p}<0.05$ ). No statistically significant differences were observed when comparing patients with controls (unpaired t-test, SPM8).

During active movement of the affected side, within-group analysis of patients showed activation of the contralateral and ipsilateral sensorimotor cortex, with the most significant clusters of activation located in the bilateral precentral gyri, contralateral superior and middle frontal gyri, supplementary motor area, contralateral inferior parietal lobule, ipsilateral insula, ipsilateral caudate body, and cerebellum (SPM8, cluster-level corrected $\mathrm{p}<0.01$; see Figure 2 and Table 3).

Table 3. Within-group analysis in patients during movement of the affected side.

\begin{tabular}{lccccc}
\hline Activation area & \multicolumn{2}{l}{ MNI Coordinates } & $\mathrm{K}$ & $\mathrm{Z}$ \\
\hline & $\mathrm{x}$ & $\mathrm{y}$ & $\mathrm{z}$ & & \\
\hline Ipsilateral superior temporal gyrus & -46 & 14 & -12 & 301 & 5.78 \\
Ipsilateral inferior frontal gyrus & -44 & 26 & -14 & 301 & 5.10
\end{tabular}




\begin{tabular}{|c|c|c|c|c|c|}
\hline & -54 & 10 & 36 & 13 & 4.89 \\
\hline Contralateral superior temporal gyrus & 48 & 8 & 2 & 172 & 5.66 \\
\hline Contralateral inferior frontal gyrus & 50 & 22 & -2 & & 5.99 \\
\hline Contralateral precentral gyrus & 58 & 12 & 10 & & 5.24 \\
\hline \multirow[t]{3}{*}{ Ipsilateral precentral gyrus } & -52 & -8 & 44 & 136 & 5.16 \\
\hline & -48 & -4 & 52 & & 4.97 \\
\hline & -50 & -10 & 36 & & 4.94 \\
\hline \multirow[t]{2}{*}{ Contralateral cerebellum } & 14 & -60 & -18 & 104 & 5.11 \\
\hline & 10 & -68 & -14 & & 4.80 \\
\hline Ipsilateral medial frontal gyrus & & -2 & 62 & 126 & 5.01 \\
\hline Contralateral medial frontal gyrus & 6 & 0 & 58 & & 4.89 \\
\hline Contralateral superior frontal gyrus & 6 & & 70 & & 4.89 \\
\hline Ipsilateral inferior parietal lobule & -54 & -26 & 24 & 19 & 4.97 \\
\hline Ipsilateral pons & -12 & -28 & -26 & 22 & 4.93 \\
\hline Ipsilateral insula & -34 & 10 & 10 & 4 & 4.85 \\
\hline Ipsilateral cerebellum & -12 & -58 & -22 & 13 & 4.82 \\
\hline
\end{tabular}

$\mathrm{MNI}=$ Montreal Neurological Institute and Hospital $\mathrm{p}<0.01 ; \mathrm{K}=$ cluster size; $\mathrm{Z}=$ peak Z-score

During active movement of the affected side, between-group analysis revealed a significantly greater activation of the ipsilateral frontal areas (inferior frontal gyrus) and the insula in patients as compared to controls (SPM8, cluster-level corrected $\mathrm{p}<0.05$; see Figure 3 and Table 4). 
Table 4. Between-group analysis of movement of the affected side in patients as compared to controls.

\begin{tabular}{lccccc}
\hline Activation area & & MNI Coordinates & $\mathrm{K}$ & $\mathrm{Z}$ \\
& $\mathrm{x}$ & $\mathrm{y}$ & $\mathrm{z}$ & \\
& & & & & \\
\hline Ipsilateral insula & -34 & 20 & -8 & 1469 & 4.09 \\
Ipsilateral inferior frontal gyrus & -48 & 16 & 2 & & 3.49 \\
& & & & & \\
\hline
\end{tabular}

$\mathrm{MNI}=$ Montreal Neurological Institute and Hospital $\mathrm{p}<0.05 ; \mathrm{K}=$ cluster size; $\mathrm{Z}=$ peak $\mathrm{Z}$-score

In patients, a significant direct correlation was observed between activation of the ipsilateral precuneus (MNI coordinates $\mathrm{x}-8, \mathrm{y}-58, \mathrm{z} 56 ; \mathrm{K} 13582, \mathrm{Z} 3.35$ ) hemisphere when moving the paretic side and the degree of paresis at baseline (SPM8, cluster-level corrected $\mathrm{p}<0.05$; see Table 5).

A significant association was also observed between activation and clinical outcome measured as complete recovery (present or absent) and as the difference between the HB score at onset and at six months, $\triangle \mathrm{HB}$ (SPM8, cluster-level corrected $\mathrm{p}<0.01$; Figures 4 and 5, Tables 6 and 7 Tables 5 and 6 ).

Table 5. Areas of significantly greater activation at baseline in patients without clinical recovery.

\begin{tabular}{lccccc}
\hline Activation area & MNI Coordinates & $\mathrm{K}$ & $\mathrm{Z}$ \\
\hline & $\mathrm{x}$ & $\mathrm{y}$ & $\mathrm{Z}$ & \\
& & & & & \\
\hline Ipsilateral precuneus & 14 & -60 & 52 & 8149 & 3.0 \\
Ipsilateral insula & 34 & 2 & 18 & & 3.0 \\
& & & & & \\
Ipsilateral supramarginal gyrus & 42 & -40 & 38 & & 2.92
\end{tabular}

$\overline{M N I}=$ Montreal Neurological Institute and Hospital $\mathrm{p}<0.01 ; \mathrm{K}=$ cluster size; $\mathrm{Z}=$ peak Z-score 
Table 6. Inverse correlation between activation of the ipsilateral hemisphere in patients when moving the paretic side and the difference between the HB score at the onset and at six months.

\begin{tabular}{|c|c|c|c|c|c|}
\hline Activation area & & dina & & $\mathrm{K}$ & $\mathrm{Z}$ \\
\hline & $\mathrm{x}$ & $\mathrm{y}$ & $\mathrm{z}$ & & \\
\hline Ipsilateral thalamus & -16 & -32 & 6 & 879 & 3.86 \\
\hline Contralateral thalamus & 2 & -6 & 6 & & 3.22 \\
\hline Ipsilateral medial frontal gyrus & -30 & 32 & 28 & 1380 & 3.79 \\
\hline Ipsilateral anterior cingulate & -12 & 20 & 26 & & 3.65 \\
\hline Ipsilateral inferior frontal gyrus & -28 & 34 & 8 & & 3.45 \\
\hline
\end{tabular}

In patients who did not experience a complete recovery we observed a greater activation of the ipsilateral hemisphere (precuneus, insula, supramarginal gyrus) when moving the paretic side (SPM8, cluster-level corrected $\mathrm{p}<0.01)$.

Between-group analysis showed no significant differences between patients and controls during passive movement of both the affected and unaffected side $(p>0.05)$. 


\section{Discussion}

The present study revealed changes in cortical activation in patients with acute Bell's palsy during active facial movement of the paretic side, whereas passive movement elicited activation patterns similar to those observed in controls. The activation pattern at baseline correlated with the degree of clinical recovery, suggesting a prognostic role of fMRI.

Facial motor function is provided by a well-known cortical network consisting of the primary motor cortex, the ventrolateral premotor cortex, and the supplementary and cingulate motor cortices. The facial component of all these cortical areas is directly connected to the facial motor nucleus via corticobulbar projections. ${ }^{28,29}$ FMRI studies have demonstrated a cortical reorganization of this network in both central and peripheral paralysis. ${ }^{16,20,21}$

According to our data, BP patients exhibited a prevalent activation of the frontal lobe and the insula of the ipsilateral hemisphere. It has been previously suggested that the ipsilateral hemisphere plays an important role in sensorimotor function. Ipsilateral pathways are known to contribute to the control of voluntary movements in normal adults. ${ }^{30}$ Hence, ipsilateral activation may also provide motor unit recruitment in patients with nervous system injury. Increased ipsilateral activation was described in patients with stroke, multiple sclerosis and vascular malformations. ${ }^{31-33}$ There are multiple theories to explain the mechanisms underlying ipsilateral motor pathway activation in patients with central and peripheral paralysis. One of the most widely accepted is the disinhibition hypothesis, which postulates that ipsilateral motor pathway activation is related to the decrease of interhemispheric transcallosal inhibition from the affected side toward the unaffected side that is caused by a structural lesion such as an ischemic infarct. The unaffected motor cortex is recruited as the origin of the ipsilateral motor pathway. ${ }^{31}$ To date, few studies have been carried out on peripheral nerve injuries. Studies conducted in rats have documented that peripheral deafferentation can produce effects similar to lesions affecting the central nervous system and that after a long period of time (from 1 week to 4 months), resection of the nerve results in the reorganization of the motor cortex, with significant activation of both ipsilateral and 
contralateral motor areas. ${ }^{34,35}$ These results suggest that deafferentation is capable of inducing changes in cortical plasticity. Ipsilateral contribution to facial movement was also documented by a transcranial magnetic stimulation study showing that stimulation to one side of the M1 cortex can cause ipsilateral motor-evoked potentials in BP patients. ${ }^{14}$

Increased ipsilateral insular and contiguous inferior frontal gyrus activation was also identified in the patient cohort. The insula may be physiologically activated during a wide array of events. The anterior and centroventral insular sector contains a mosaic of orofacial motor programs involved in facial movements that are likely associated with the well-known insular gustatory and visceromotor representations. A sensorimotor field occupies the caudal-dorsal portion of the insula and appears as an extension of the parietal lobe. ${ }^{36}$ The inferior frontal gyrus is generally recruited when important cues are detected, regardless of whether that detection is followed by the inhibition of a motor response, the generation of a motor response, or no external response at all. ${ }^{37} \mathrm{We}$ postulate that due to intact sensory feedback, BP patients perceive that the movement they are performing is not correctly executed, which leads to the recruitment of the insula for facial movement execution and the inferior frontal gyrus for motor control in order to compensate for the inefficiency.

In this study, the facial motor area response differed according to whether the task was passive or active. Previous studies on patients with stroke have demonstrated that passive movement activates areas similar to those elicited during active movement in central paralysis. ${ }^{38}$ Differences in activation during passive or active movement in BP patients may be due to the integrity of the sensory afferent in $\mathrm{BP}$, which is usually not the case in stroke. The facial nerve and the associated nerve pathways are almost exclusively motor: the afferent sensorimotor face fiber is conveyed by the trigeminal nerve. During passive movement, the sensory cortex conveys the correct execution of the movement as it occurs in the absence of paresis. During active movement, the sensory input records the ineffectiveness of the motor output, leading to an increase or change in activation.

Our results revealed a correlation between the degree of activation and clinical outcome. We demonstrated a significant correlation between the activation of the ipsilateral precuneus and symptom severity at onset and between the activation of the ipsilateral precuneus, insula, and supramarginal gyrus 
and clinical outcome. These areas are major components of executive control functions and are implicated in the control of conscious processes and the modulation of actions. ${ }^{39,40}$ It is conceivable that BP patients with higher degrees of nerve damage and worse motor performance might need a greater contribution from areas involved in error correction and action control.

The precuneus plays an essential role in self-processing operations and visuospatial imagery. ${ }^{39}$ It is activated during movement imagery more than during movement execution ${ }^{41}$, and it is also strongly involved in the mental rendering of images held in working memory. ${ }^{42} \mathrm{~A}$ previous work on BP and fMRI during facial movement hypothesized that the difficulty in correctly executing the task leads to activation of the image of the intended movement to sustain the performance. ${ }^{20}$ Indeed, the supramarginal gyrus is involved in the suppression of the prepared but incorrect response and its replacement with a new appropriate action..$^{40}$

The main limitation of the present study was the inability to obtain clinical information of patients at the one-year follow-up, which could have been used to better define fMRI correlates to residual palsy, such as paresis and synkinesis. Furthermore, the monitoring of unaffected/affected side facial movements was carried out by visual inspection. Future studies may use electromyographic recording to monitor muscle side involvement for a more objective assessment, as well as electroneurography signs of denervation for a more precise determination of correlation with palsy outcomes.

This study has demonstrated cortical plasticity functional changes in patients suffering from Bell's palsy, mainly consisting in an increased involvement of ipsilateral motor cortex in the early phase following facial palsy. These changes are associated with clinical status both at baseline and at follow-up suggesting that fMRI may represent a useful tool to predict long-term outcome in patients affected by Bell's palsy, establish the correct therapeutic strategy, and monitor treatment efficacy. 


\section{References}

1. Peitersen E. Bell's palsy: the spontaneous course of 2,500 peripheral facial nerve palsies of different etiologies. Acta Otolaryngol 2002;549:4s-30s.

2. Yanagihara N. Incidence of Bell's palsy. Ann Otol Rhinol Laryngol 1988; 137:3s-4s.

3. Engström M, Berg T, Stjernquist-Desatnik A, et al. Prednisolone and valaciclovir in Bell's palsy: a randomised, double-blind, placebo-controlled, multicentre trial. Lancet Neurol 2008;7:9931000.

4. Gantz BJ, JRubinstein JT, Gidley P, Woodworth GG. Surgical management of Bell's palsy. Laryngoscope 1999;109:1177-88.

5. Nicastri M, Mancini P, De Seta D, et al. Efficacy of early physical therapy in severe Bell's palsy: a randomized controlled trial. Neurorehabil Neural Repair 2013;27:542-51.

6. Beurskens CH, Oosterhof J, Nijhuis-van der Sanden MW. Frequency and location of synkineses in patients with peripheral facial nerve paresis. Otol Neurotol 2010;31:671-5.

7. Van Amstel AD, Devriese PP. Clinical experiences with recurrences of Bell's palsy. Arch Otorhinolaryngol 1988;245:302-6.

8. Linder TE, Abdelkafy W, Cavero-Vanek S. The management of peripheral facial nerve palsy: paresis versus paralysis and sources of ambiguity in study designs, Otol Neurotol 2010;31:319_ 27.

9. Mantsopoulos K, Psillas G, Psychogios G. Predicting the long-term outcome after idiopathic facial nerve paralysis. Otol Neurotol 2011;32:848-51.

10. Berg T, Bylund N, Marsk E et al. The effect of prednisolone on sequelae in Bell's palsy. Arch Otolaryngol Head Neck Surg 2012; 138:445-9.

11. Fisch U. Prognostic value of electrical tests in acute facial paralysis. Am J Otol 1984;5:494-98. 
12. Sittel C, Stennert E. Prognostic value of electromyography in acute peripheral facial nerve palsy. Otol Neurotol 2001;22:100-4.

13. Mancini P, De Seta D, Prosperini L, et al. Prognostic factors of Bell's palsy: multivariate analysis of electrophysiological findings. Laryngoscope 2014;124:2598-605.

14. Rijntjes M, Tegenthoff M, Liepert J et al. Cortical reorganization in patients with facial palsy. Ann Neurol 1997; 41:621-30.

15. Yildiz S, Bademkiran F, Yildiz N et al. Facial motor cortex plasticity in patients with unilateral peripheral facial paralysis. NeuroRehabilitation 2007;22:133-40.

16. Ward NS, The neural substrates of motor recovery after focal damage to the central nervous system. Arch Phys Med Rehabil 2006;87:S30-S35.

17. Loubinoux I, Dechaumont-Palacin S, Castel-Lacanal E, et al. Prognostic value of FMRI in recovery of hand function in subcortical stroke patients. Cereb Cortex 2007;17:2980-7.

18. Caramia F, Mainero C, Gragnani F, et al. Functional MRI changes in the central motor system in myotonic dystrophy type 1. Magn Reson Imaging 2010;28:226-34.

19. Klingner CM, Volk GF, Maertin A, et al. Cortical reorganization in Bell's palsy. Restor Neurol Neurosci 2011;29:203-14.

20. Klingner CM, Volk GF, Brodoehl S, et al. Time course of cortical plasticity after facial nerve palsy: a single-case study, Neurorehabil Neural Repair 2012;26:197-203.

21. Song W, Dai M, Xuan L, et al. Sensorimotor cortical neuroplasticity in the early stage of Bell's palsy. Neural Plast 2017;2017:8796239

22. Lee J, Yang J, Li C, et al. Cortical reorganization in patients recovered from Bell's palsy: an orofacial and finger movements task-state fMRI study. Neural Plast 2016;2016:8231726. 
23. Song W, Cao Z, Lang C, et al. Disrupted functional connectivity of striatal sub-regions in Bell's palsy patients. Neuroimage Clin 2017;14:122-9.

24. Han X, Li H, Du L, et al. Differences in functional brain alterations driven by right or left facial nerve efferent dysfunction: Evidence from early Bell's palsy. Quant Imaging Med Surg 2019;9:427-39.

25. House JW, Brackmann DE. Facial nerve grading system. Otolaryngol Head Neck Surg 1985;93:146-7.

26. Oldfield RC. The assessment and analysis of handedness: the Edinburgh inventory. Neuropsychologia 1971;9:97-113.

27. Bendet E, Vajtai I, Maranta C, Fisch U. Rate and extent of early axonal degeneration of the human facial nerve. Ann Otol Rhinol Laryngol 1998;107:1-5.

28. Morecraft RJ, Stilwell-Morecraft KS, Rossing WR. The motor cortex and facial expression: new insights from neuroscience. Neurologist 2004;10:235-49.

29. Hesselmann V, Sorger B, Lasek K, et al. Discriminating the cortical representation sites of tongue and up movement by functional MRI. Brain Topogr 2004;16:159-67.

30. Kobayashi M, Hutchinson S, Schlaug G, Pascual-Leone A. Ipsilateral motor cortex activation on functional magnetic resonance imaging during unilateral hand movements is related to interhemispheric interactions. Neuroimage 2003;20:2259-70.

31. Jang SH. A review of the ipsilateral motor pathway as a recovery mechanism in patients with stroke. NeuroRehabilitation 2009;24:315-20.

32. Zeller D, Dang SY, Stefan K et al. Functional role of ipsilateral motor areas in multiple sclerosis, J Neurol Neurosurg Psychiatry 2011;82:578-83. 
33. Caramia F, Francia A, Mainero C, et al. Neurophysiological and functional MRI evidence of reorganization of cortical motor areas in cerebral arteriovenous malformation. Magn Reson Imaging 2009;27:1360-9.

34. Pelled G, Bergstrom DA, Tierney PL et al. Ipsilateral cortical fMRI responses after peripheral nerve damage in rats reflect increased interneuron activity. Proc Natl Acad Sci USA 2009;106:14114-9.

35. Sanes JN, Suner S, Donoghue JP. Dynamic organization of primary motor cortex output to target muscles in adult rats. I. Long-term patterns of reorganization following motor or mixed peripheral nerve lesions Exp Brain Res 1990;79:479-91.

36. Jezzini A, Caruana F, Stoianov I, Gallese V, Rizzolatti G. Functional organization of the insula and inner perisylvian regions. Proc Natl Acad Sci USA 2012; 109:10077-82.

37. Hampshire A, Chamberlain SR, Monti MM, Duncan J, Owen AM. The role of the right inferior frontal gyrus: inhibition and attentional control. Neuroimage 2010; 50:1313-9.

38. Tombari D, Loubinoux I, Pariente J et al. A longitudinal fMRI study: in recovering and then in clinically stable sub-cortical stroke patients. Neuroimage 2004;23:827-39.

39. Cavanna AE, Trimble M. The precuneus: a review of its functional anatomy and behavioural correlates. Brain 2006; 129:564-83.

40. Hartwigsen G, Bestmann S, Ward NS, et al. Left dorsal premotor cortex and supramarginal gyrus complement each other during rapid action reprogramming. J Neurosci 2012;32:16162-71.

41. Hanakawa T, Immisch I, Toma K et al. Functional properties of brain areas associated with motor execution and imagery. J Neurophysiol 2003;89:989-1002.

42. Addis DR, McIntosh AR, Moscovitch M, Crawley AP, McAndrews MP. Characterizing spatial and temporal features of autobiographical memory retrieval networks: a partial least squares approach. Neuroimage 2004; 23:1460-71. 

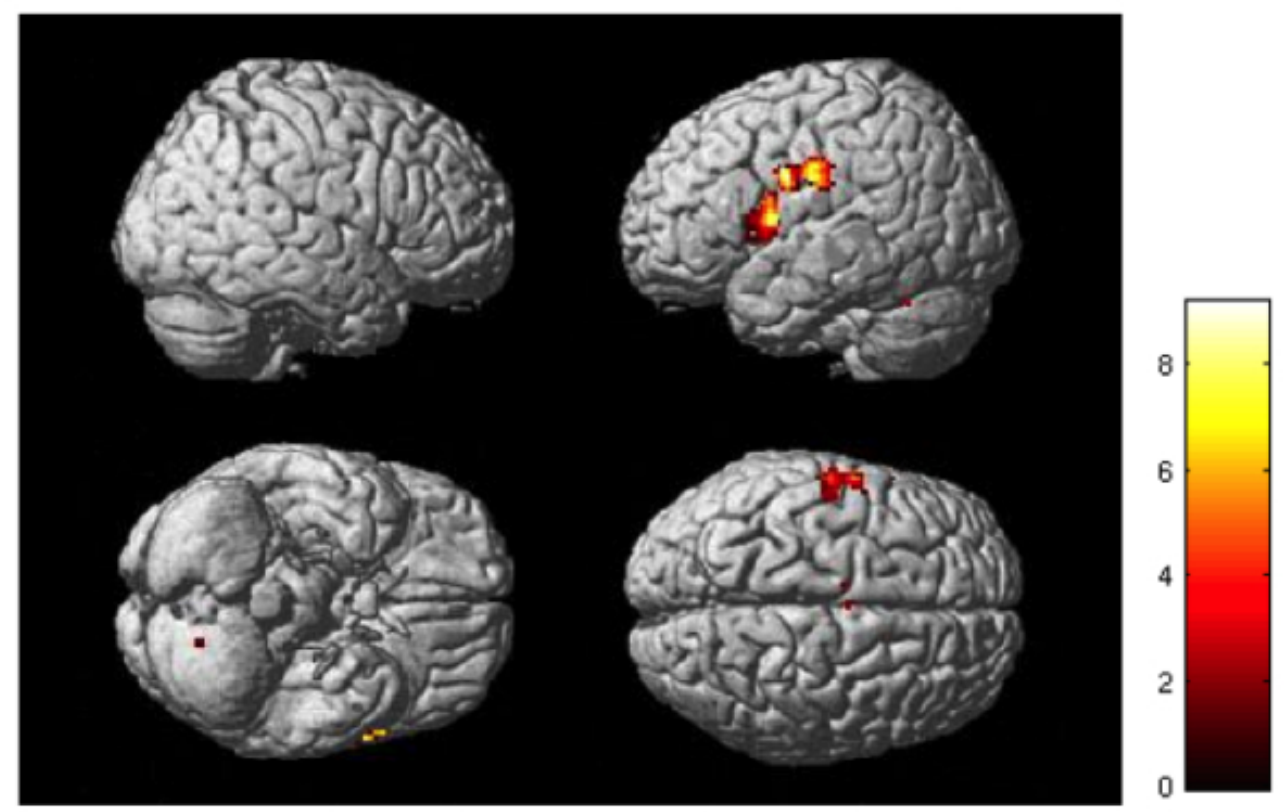

Fig 1. Within-group analysis of controls during active motor tasks. During active motor tasks, within-group analysis of controls showed activation of areas located on the hemisphere contralateral to the movement and in the bilateral cerebellum (contralateral precentral gyrus and inferior frontal gyrus, contralateral postcentral gyrus and medial frontal gyrus, bilateral cerebellum) (SPM8, cluster-level corrected $p<0,05)$. $\mathrm{L}=$ left; $\mathrm{R}=$ right. Color bar displays the $\mathrm{z}$ score.

$211 \times 136 \mathrm{~mm}(72 \times 72 \mathrm{DPI})$ 

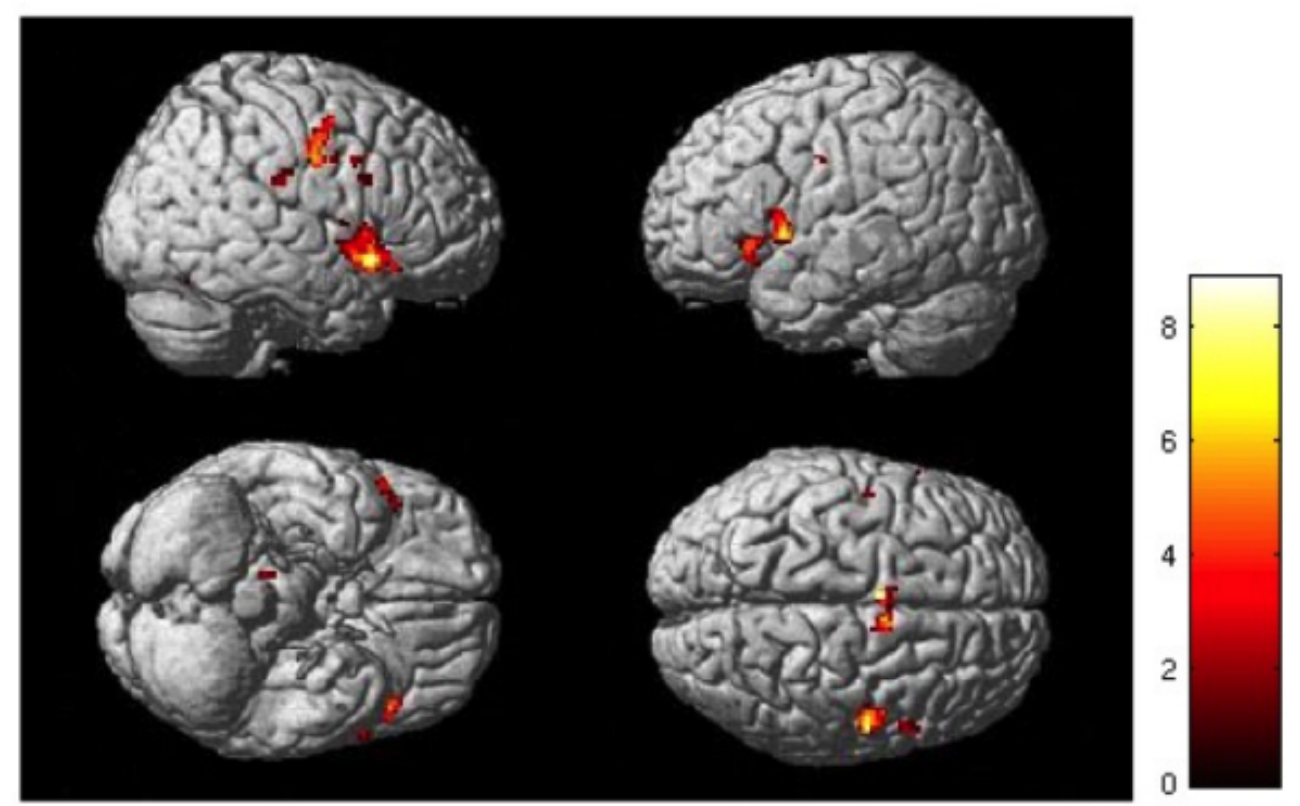

2. Within-group analysis of patients during movement of the affected side. During active movement of the affected side, within-group analysis of patients showed activation of the contralateral and ipsilateral sensorimotor cortex, with the most significant clusters of activation located in the bilateral precentral gyri, contralateral superior and middle frontal gyri, supplementary motor area, contralateral inferior parietal lobule, ipsilateral insula, ipsilateral caudate body, and cerebellum (SPM8, cluster-level corrected p <0,05). $\mathrm{L}=$ left; $\mathrm{R}=$ right. Color bar displays the $\mathrm{z}$ score.

\section{$211 \times 140 \mathrm{~mm}(72 \times 72 \mathrm{DPI})$}



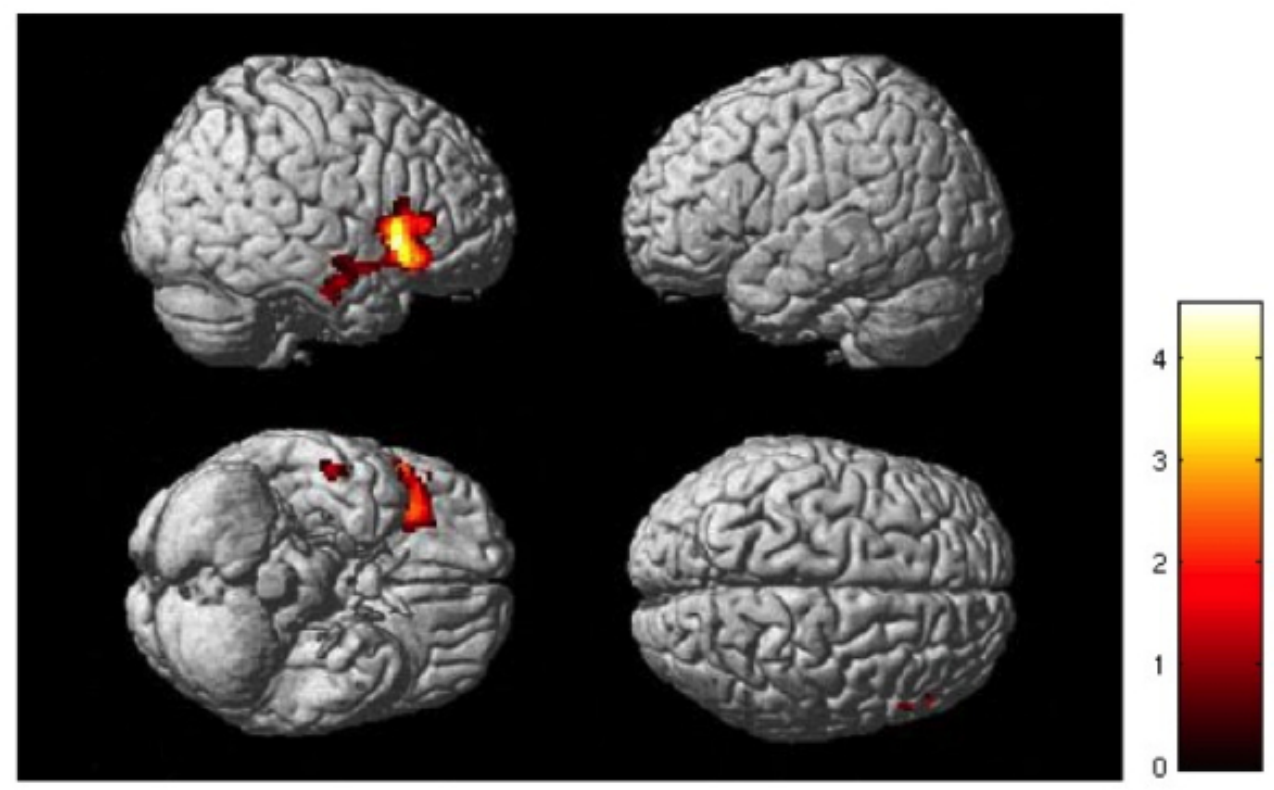

3. Between-group analysis during movement of the affected side. During active movement of the affected side, between-group analysis revealed a significantly greater activation of the ipsilateral frontal areas (inferior frontal gyrus) and the insula in patients as compared to controls (SPM8, cluster-level corrected $p$ $<0,05)$. $L=$ left; $R=$ right. Color bar displays the $z$ score. 

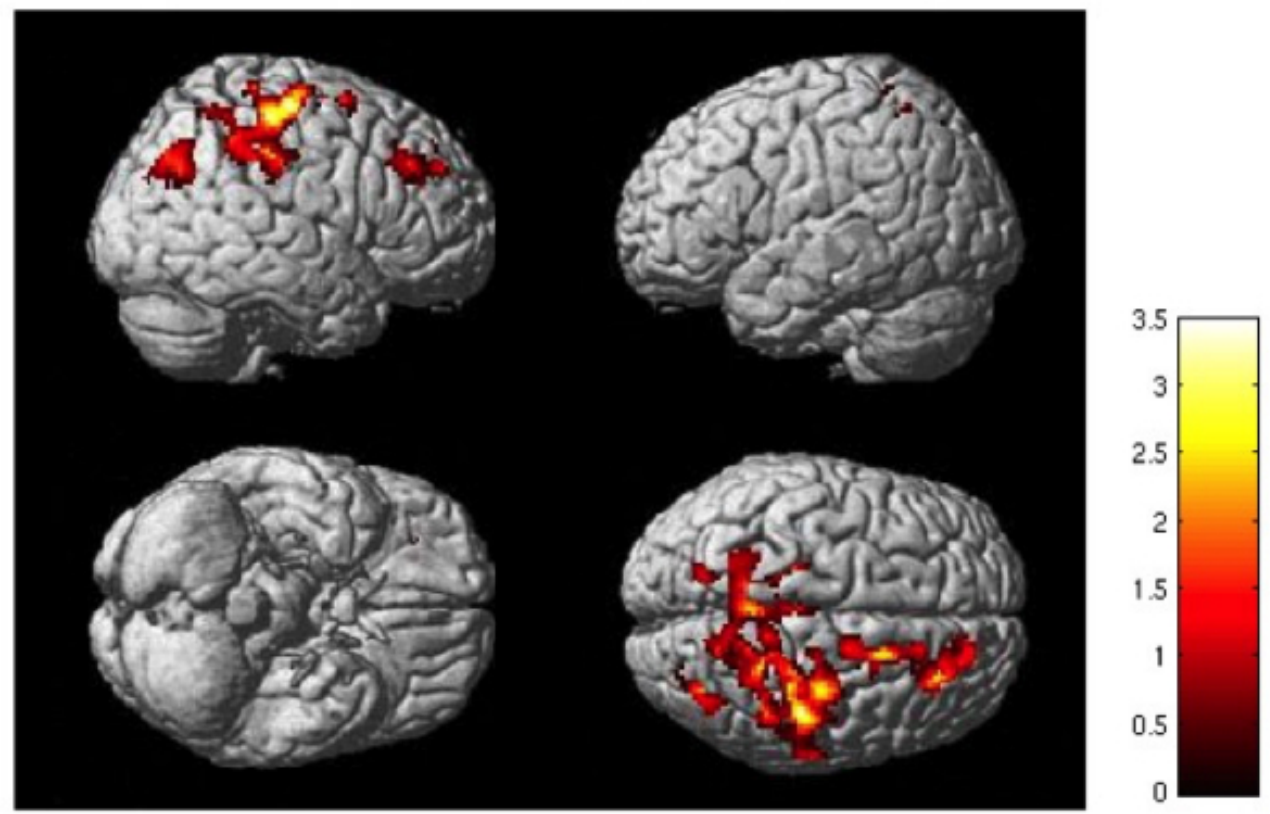

Fig 4. Differences in activation between patients with and without clinical recovery. Greater activation of the ipsilateral hemisphere when moving the paretic side was seen in patients who did not experience a complete recovery (SPM8, cluster-level corrected $p<0,01)$. $L=$ left; $R=$ right. Color bar displays the $z$ score.

$211 \times 137 \mathrm{~mm}(72 \times 72 \mathrm{DPI})$ 

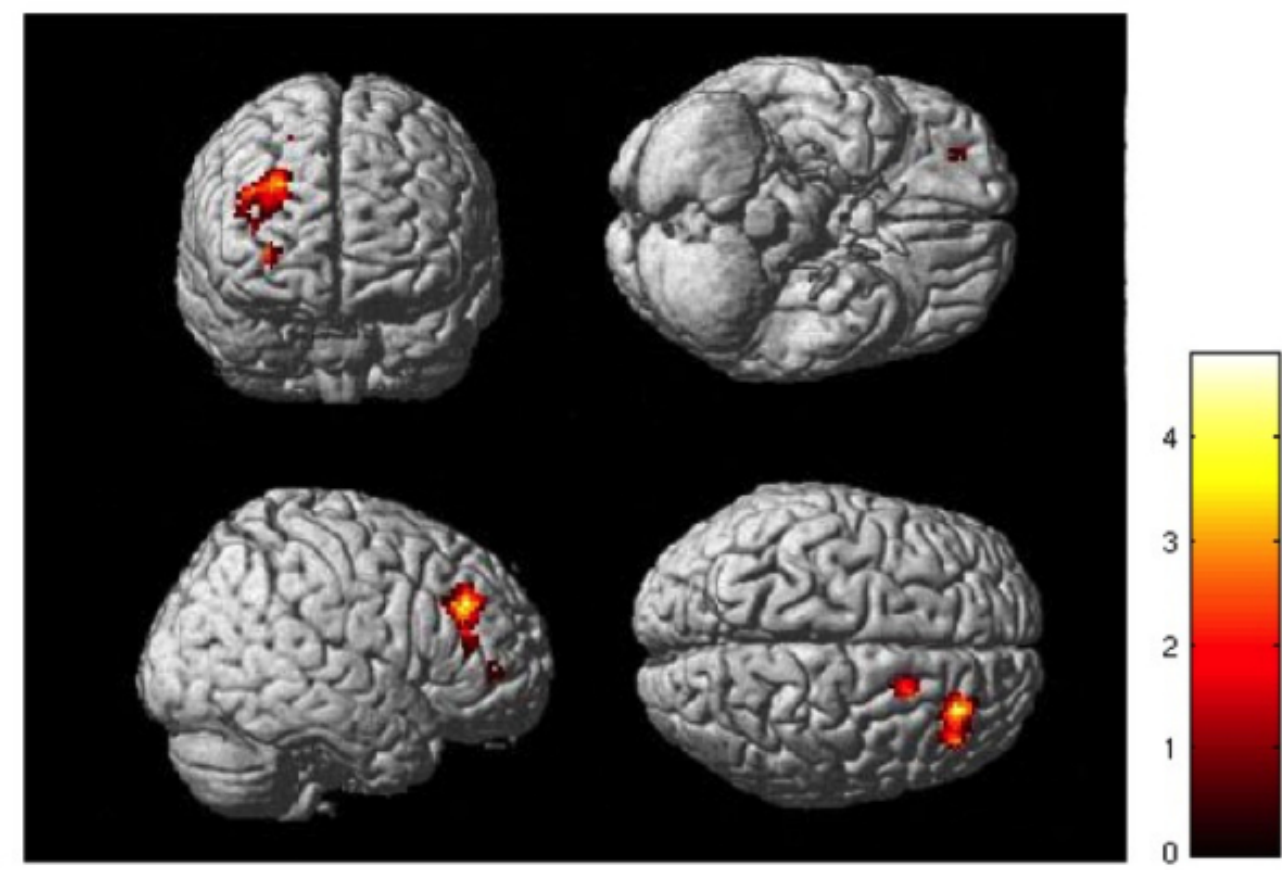

Fig 5. Inverse correlation between activation and $\triangle \mathrm{HB}$. Inverse correlation between activation of the ipsilateral hemisphere in patients when moving the paretic side, and the difference between the $\mathrm{HB}$ score at onset and at six months $(\Delta \mathrm{HB})$, revealing a significant association (SPM8, cluster-level corrected $\mathrm{p}<0,01$ ). $\mathrm{L}=$ left; $\mathrm{R}=$ right. Color bar displays the $\mathrm{z}$ score.

$211 \times 145 \mathrm{~mm}(72 \times 72 \mathrm{DPI})$ 